\title{
Josué de Castro : actualité d'une pensée
}

\author{
Magda Zanoni \\ Écologue et sociologue, UMR7533 LADYSS, CNRS, Université Paris Ouest Nanterre La Défense, bâtiment K, \\ 200 avenue de la République, 92001 Nanterre cedex, France
}

\begin{abstract}
Au début de l'année 2009, s'est tenu à l'Université Paris 8 (Saint-Denis) un colloque intitulé « Josué de Castro dans le XXI siècle ${ }^{1}$ ». Cette initiative voulait, certes, être un hommage rendu à la personnalité exceptionnelle de l'homme à l'occasion du centenaire de sa naissance $(1908)^{2}$. Mais il s'agissait avant tout, comme le souligne l'intitulé du colloque, de se demander comment sa pensée et son œuvre, qui s'inscrivent pour l'essentiel dans la première moitié du XX $X^{\mathrm{e}}$ siècle ${ }^{3}$, peuvent aider à comprendre notre monde contemporain. Il est significatif que l'hommage qui lui a fait défaut lorsqu'il est décédé (1973) lui soit rendu en ce début du XXI siècle : c'est que les questions auxquelles il a consacré son énergie (la faim et le sous-développement), loin d'être du passé, sont toujours lancinantes ${ }^{4}$; mais c'est aussi - et sans doute davantage encore - que la façon originale qu'il avait de les aborder a besoin d'être rappelée. Il est, en effet, plus que jamais indispensable de s'en imprégner et de se demander s'il ne serait pas pertinent de s'en inspirer pour sortir des ornières qui conduisent à faire perdurer le passé. C'est donc une pensée pionnière que l'Université Paris 8 voulait faire revivre, et ce, pour les besoins du présent. La tâche lui revenait de plein droit, puisque - symbole s'il en est ${ }^{5}$ ! c'est cette institution qui l'avait accueilli en tant que professeur associé en 1968, alors qu'il avait dû choisir l'exil après le coup d'État militaire qui s'était produit au Brésil en $1964^{6}$
\end{abstract}

La Rédaction

Les origines de Josué de Castro expliquent sans doute pour beaucoup qu'il ait consacré sa vie, tant sur le plan professionnel que sur le plan personnel, à lutter contre

Auteur correspondant : magdazanoni@gmail.com

${ }^{1}$ Ce colloque était organisé par le LADYSS (UMR CNRS / Universités Paris 10, Paris 1, Paris 7, Paris 8) sous la responsabilité d'Alain Bué, maître de conférence, et de Françoise Plet, professeur (département de géographie de l’Université Paris 8).

2 Un autre colloque, organisé par le Centre Josué de Castro, a eu lieu en novembre 2008, à Recife.

${ }^{3}$ Les deux ouvrages qui lui ont donné une audience internationale datent l'un (Géographie de la faim) de 1946 et l'autre (Géopolitique de la faim) de 1951.

${ }^{4}$ Les récentes émeutes de la faim, consécutives à la hausse globale des prix de l'alimentation, doublées par la crise généralisée qui pénalise d'abord les plus pauvres, ont remis au centre des préoccupations internationales les questions fondamentales de l'accès de tous à une nourriture suffisante et équilibrée.

${ }^{5}$ Pur produit des événements et de l'esprit de 1968, l'Université Paris 8 était alors située à Vincennes et portait le nom de Centre universitaire expérimental de Vincennes, qui soulignait combien elle dérogeait aux règles de l’Université classique.

${ }^{6}$ Il est décédé à Paris en septembre 1973, après s'être vu refuser l'autorisation de rentrer au Brésil, qu'il avait sollicitée pour raisons de santé. Le gouvernement militaire a néanmoins donné l'autorisation du transfert de son corps. la malnutrition et le sous-développement. Il est, en effet, natif de la région semi-aride du Nordeste brésilien (le Sertão), pays de la pauvreté et de la faim dominé par le latifundium. Son père et sa mère étaient l'un et l'autre originaires de la région. Ils étaient propriétaires fonciers et vivaient du commerce du bétail. La grande sécheresse de 1877 contraignit la famille à quitter la région pour s'installer à Recife, capitale de l'un des États du Nordeste, le Pernambouc. C'est là que Josué de Castro naquit, le 5 septembre 1908.

Sa personnalité comportait deux facettes étroitement liées l'une à l'autre : celle d'un chercheur qui puisait dans son engagement social et politique son inspiration novatrice; celle d'un citoyen et homme politique qui puisait dans son travail de chercheur les raisons et les arguments de son action. Ses idées inspirent toujours de nombreuses recherches sur l'alimentation et la sousalimentation, et ce, dans différents domaines ${ }^{7}$. Elles sont à la base des politiques publiques brésiliennes destinées à l'agriculture familiale et à la réforme agraire.

\footnotetext{
${ }^{7}$ Les contributions au colloque "Josué de Castro dans le XXI ${ }^{\mathrm{e}}$ siècle » ont abondamment illustré cette permanence de son influence. La publication des actes est prévue en 2010.
} 


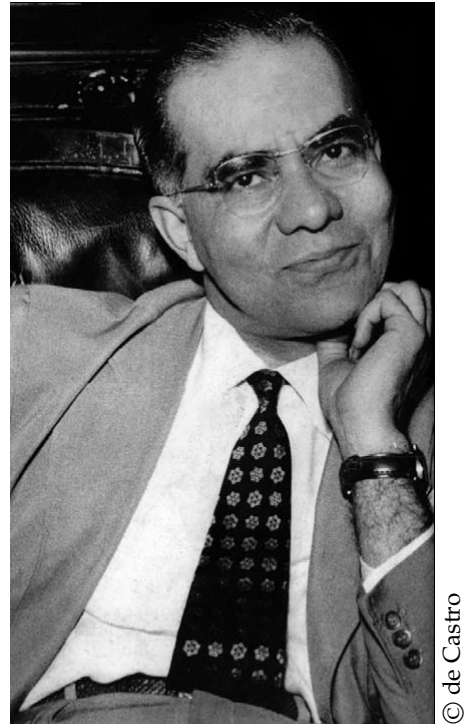

Josué de Castro

\section{Un pionnier de la démarche interdisciplinaire}

L'itinéraire de Josué de Castro est à lui seul tout à fait instructif. Après des études primaires et secondaires à Recife, il est admis à la faculté de médecine de l'Université du Brésil (Rio de Janeiro) et obtient son diplôme en 1929 (il a alors 21 ans). De retour dans sa ville natale, il exerce en tant que médecin endocrinologue. Il assure en même temps un enseignement de physiologie à la faculté de médecine. En 1932, il soutient sa thèse de LivreDocência portant sur les problèmes physiologiques qui découlent de la sous-alimentation au Brésil (Encadré 1$)^{8}$. Une période féconde s'ensuit, où il mène des recherches sur les conditions de vie et de travail des ouvriers dans la ville de Recife et sur celles des travailleurs en milieu rural dans le Pernambouc, toujours en rapport avec l'alimentation. Cela donne lieu à la publication d'un ouvrage. Il acquiert ainsi très rapidement une renommée nationale, ce qui lui vaut une invitation à l'Université du district fédéral de Rio de Janeiro, puis à l'Université du Brésil (devenue postérieurement Université fédérale de Rio de Janeiro [UFRJ]).

Il se produit alors un basculement décisif dans ses orientations intellectuelles. Sans abandonner ses enseignements sur les questions de nutrition et d'alimentation à la faculté nationale de médecine, il opte définitivement pour l'intégration d'une approche géographique dans ses analyses de la faim, de l'alimentation et des conditions de vie des populations. Rattaché à la chaire de géographie humaine, il soutient une thèse sur la ville de Recife et sur les facteurs qui ont déterminé sa localisation. Il devient

\footnotetext{
8 Pour les publications afférentes aux autres recherches citées
} dans ce texte, voir aussi cet encadré.
Encadré 1. Principaux ouvrages ${ }^{a}$

- O problema da alimentação no Brasil, São Paulo/Rio de Janeiro, Companhia Editôra Nacional, 1933. Trad. fr. : Le Problème de l'alimentation en Amérique du Sud, Paris, Dunod, 1950.

- Condições de vida das classes operárias do Recife, Recife, Departamento de Saúde Pública, 1935.

- A questão do salário minimo, Rio de Janeiro, Ministério do Trabalho, Industria e Comércio, 1935.

- Alimentação e raça, Rio de Janeiro, Editôra Civilização Brasileira, 1935.

- A alimentação brasileira à luz da geografia humana, Pôrto Alegre, Livraria do Globo, 1937.

- Fisiologia dos tabus, Rio de Janeiro, Editôra Nestlé, 1939.

- Geografia da fome, Rio de Janeiro, Editôra O Cruzeiro, 1946.

Trad. fr. : Géographie de la faim, Paris, Éditions ouvrières Économie et Humanisme, 1949.

- Fatores de localização da cidade do Recife, Rio de Janeiro, Imprensa nacional, 1948.

- Geopolítica da fome, Rio de Janeiro, Livraria-Editôra da Casa do Estudante do Brasil, 1951. Trad. fr. : Géopolitique de la faim, Paris, Éditions ouvrières Économie et Humanisme, 1952.

- Sete palmos de terra e um caixão : ensaio sobre o Nordeste, área explosiva, São Paulo, Editôra Brasiliense, 1965. Trad. fr. : Une zone explosive, le Nordeste du Brésil, Paris, Le Seuil, 1965.

- O livro negro da fome, São Paulo, Editôra Brasiliense, 1957. Trad. fr. : Le Livre noir de la faim : manifeste pour la création d'une association mondiale de lutte contre la faim, Paris, Éditions ouvrières Économie et Humanisme, 1957.

- Homens e caranguejos, São Paulo, Editôra Brasiliense, 1967. Trad. fr. : Des hommes et des crabes, Paris, Le Seuil, cop. 1966.

Cette bibliographie a été établie à partir de celle réalisée par Marion Corbin pour le colloque «Josué de Castro dans le XXI ${ }^{e}$ siècle ». Elle ne mentionne que les premières éditions, aussi bien pour les textes originaux que pour leur traduction française.

professeur titulaire de la chaire de géographie humaine en 1957.

Dès les premières années de sa carrière, il s'est soucié de diffuser les connaissances et de les appliquer. Entre 1939 et 1941, il met en place le Service central d'alimentation du Brésil. En 1942, il dirige le Service technique d'alimentation qui vient d'être créé par le gouvernement et réorganise l'approvisionnement alimentaire. En 1946, il devient directeur de l'Institut de nutrition de l'Université du Brésil. Cette même année, il publie Géographie de la faim et, en 1951, Géopolitique de la faim, ce qui lui assure une dimension internationale qui ne devait pas se démentir (Encadré 2).

\section{Une alternative à la «pulvérisation des savoirs »}

Son grand apport fut donc sa façon nouvelle d'appréhender la question de l'alimentation. Selon lui, en l'abordant sous des aspects à chaque fois partiels, les études n'en donnent qu'une vision tronquée. Seule une approche 


\section{Encadré 2. Distinctions et fonctions : une sélection}

- 1946 : prix José Veríssimo, Académie brésilienne des lettres. - 1952-1956 : président de la FAO.

- 1957 : fondateur et président de l'Association mondiale de lutte contre la faim (ASCOFAM).

- 1960 : président de la Campagne de lutte contre la faim promue par l'ONU.

- 1962-1964 : ambassadeur du Brésil auprès de l'ONU à Genève.

- 1963 et 1970 : nominé au prix Nobel de la paix.

- 1964 : Prix international de la paix décerné par le Conseil mondial de la paix.

d'ensemble permettrait de comprendre le problème de la sous-nutrition et de prévoir des solutions adéquates, d'où sa critique des « spécialistes » (Encadré 3).

Il envisage donc une nouvelle perspective de recherche prenant simultanément en considération l'ensemble des aspects du problème, c'est-à-dire ses dimensions tant économiques et sociales que biologiques. Il adopte déjà une approche systémique dans laquelle sont soulignées les connexions entre les multiples facteurs qui interviennent dans la construction d'un phénomène complexe.

Ainsi, lorsqu'il décrit la zona da mata (partie du Nordeste couverte par la forêt tropicale), il part d'une analyse des spécificités de l'écosystème initial - sa biodiversité, son fonctionnement - pour comprendre les perturbations écologiques provoquées au XVIe siècle par les déboisements et l'introduction de la culture de la canne à sucre dus à la colonisation portugaise. Il est de cette façon conduit à accorder une attention particulière aux interactions entre l'homme, la technique et le milieu. Il s'attache à montrer comment la culture de la canne provoque l'érosion des sols, la perte de leur fertilité, la réduction de la biodiversité végétale et animale et comment ces dégradations des ressources et des milieux sont en rapport direct avec la sous-alimentation des populations.

Mais pour bien comprendre comment ces détériorations ont pu avoir lieu, il est nécessaire de parler de la structure foncière qui s'est mise en place et qui les a causées, le latifundium. Or, évoquer ce dernier, c'est aussi faire référence à une organisation sociale inégalitaire, fondée sur une classe dominante esclavagiste qui ne se reproduit que par la destruction de la forêt et par l'exploitation du travail esclave. Ainsi, la sous-nutrition et la faim qui frappent ces populations sont le fait d'une étroite conjonction entre deux facettes intrinsèquement liées d'un système social : ses effets sur les ressources naturelles et son caractère inégalitaire. Ce panorama explicatif dressé, Josué de Castro peut retrouver sa formation initiale en abordant l'analyse des aspects sanitaires de la sous-nutrition. Cela lui donne l'occasion d'apporter une contribution essentielle aux connaissances sur les carences nutritives des populations

\section{Encadré 3. La « civilisation des spécialistes »}

«Cette limitation du champ de vision constitue également un trait caractéristique de la civilisation occidentale. Depuis le milieu du XIX ${ }^{\mathrm{e}}$ siècle, nous voyons se développer chez nous un type d'enseignement universitaire qui ne se préoccupe plus de transmettre une image globale du monde, mais se propose de fournir une série de clichés de ses réalités partielles, didactiquement mutilées dans l'intérêt prétendu de chacune des sciences. Le formidable choc du progrès scientifique a fini par fragmenter la culture, par la pulvériser en petits grains de savoir. Chaque spécialiste de la science s'agrippe de toutes ses forces à son grain de poussière, le tourne et le retourne sous la puissante lentille de son microscope pour en pénétrer le microcosme avec un dédain stupéfiant et une ignorance crasse pour tout ce qui se passe alentour. Dans les universités européennes et nord-américaines, on a, de notre temps, développé à l'extrême ce type d'enseignement qui vise à la spécialisation et crée - à l'intérieur de la culture, un type sui generis de civilisation - la civilisation des spécialistes, qui est dirigée par des hommes doués d'une vision technique rigoureuse, mais affligés d'une déplorable myopie culturelle et politique. C'est avec raison que Ortega y Gasset affirme que ce genre d'enseignement universitaire est responsable de la formation de "nouveaux barbares, hommes toujours plus savants, mais toujours plus incultes" (Ortega y Gasset, El libro de las Misiones, 1940). Et, chose plus grave encore, ces nouveaux barbares constituent le type dominant de nos élites intellectuelles et représentent le processus de dynamique sociale qui a provoqué ce que Rathenau appelle très justement "l'invasion verticale des barbares". Ces spécialistes limités, "qui savent toujours plus de toujours moins", sont des produits hautement dangereux pour la vie de la culture. Si la société de consommation, produit d'un type de culture mutilant l'intelligence et l'imagination humaines, est aujourd'hui partout en contestation ${ }^{a}$, c'est qu'on a compris qu'elle ne pourrait permettre un authentique bien-être social des groupes humains en satisfaisant les besoins du corps et de l'esprit. » (Castro, J. de, 1971. Géopolitique de la faim, Paris, Éditions ouvrières Économie et Humanisme, p. 42.)

Il faut souligner que ces lignes ont été écrites en 1950 !

(avitaminoses, déficits protéiques, maladies endémiques et épidémiques, etc.). Ces carences, du fait de l'exode rural que provoque le système social en place, touchent aussi bien les populations urbaines que les populations rurales. Ce cheminement allant de l'écologie aux sciences sociales lui permet de dresser le constat de faillite du système de la monoculture de la canne à sucre.

En tant que géographe, il s'est inscrit dans le courant français de la géographie régionale, alors très influente au Brésil. Cette orientation - dont il convient de souligner le caractère interdisciplinaire - lui paraissait susceptible d'apporter « une nouvelle et féconde vitalité à la vieille science [géographique] dont la démarche ne prenait guère en compte la réalité et la singularité des différents paysages vivants du monde; plus descriptive 
$q^{\prime}$ 'interprétative, plus morte que vivante ${ }^{9} »$. La méthode géographique qu'il qualifiait d'interprétative consiste à donner la priorité à la réalité géographique, dans des espaces précis. C'est en effet ce qui permet d'éviter que l'analyse ne soit noyée dans des généralités, d'utiliser la méthode comparative pour mettre en évidence les contraintes propres à chaque situation, de bien identifier et mettre en rapport de façon affinée les phénomènes naturels et sociaux. Tout cela, en vue de pouvoir remonter aux causes du problème étudié et donc d'en fournir une analyse critique.

\section{Un précurseur de l'écologie humaine}

Ce choix de l'échelle de la région et d'une géographie des relations entre milieux physiques et sociétés humaines le met en mesure d'analyser in situ les destructions des richesses naturelles, les déséquilibres écologiques provoqués par l'homme et même les violences faites localement aux populations et à leurs cultures. Cellesci, en effet, sont dues, selon lui, à une insuffisante prise en considération des interactions entre le naturel et le social, qui constituent les bases des réalités géographiques locales. La justesse de cette analyse saute aux yeux quand on regarde les conséquences d'une application brutale $\mathrm{du}$ principe «penser globalement, agir localement».

En se situant dans cette perspective, Josué de Castro est conduit à inclure de plus en plus l'écologie dans sa démarche. Dans l'introduction de son ouvrage Géographie de la faim, il prétend contribuer à un «essai de nature écologique». En réalité, que fait-il ? Il analyse les habitudes alimentaires de différents groupes humains liés à des aires géographiques déterminées. Dans cette approche, il cherche à identifier les causes multiples (naturelles et sociales) des régimes alimentaires régionaux et des manques qui les caractérisent sur le plan nutritionnel. Partant de là, il cherche à établir jusqu'où ces carences peuvent influencer la structure économico-sociale des populations étudiées ${ }^{10}$. Il est clair que cette démarche se situe au croisement d'une anthropologie et d'une écologie de la nutrition humaine : on peut dire qu'elle est à proprement parler une écologie humaine avant la lettre. De fait, vingt années après avoir écrit ces lignes (qui datent de 1946, année de la première édition de Géographie de la faim), Josué de Castro sera l'initiateur, dans le département de géographie de l'Université Paris 8 , d'un cursus d'écologie humaine...

Un autre exemple allant dans ce sens est fourni par la recherche qu'il a faite, toujours dans le Nordeste brésilien,

\footnotetext{
9 Castro, J. de, 1965. Geografia da fome, São Paulo, Editôra Brasiliense [ $1^{\text {re }}$ éd. Rio de Janeiro, Editôra O Cruzeiro], p. 240 Toutes les citations issues des ouvrages en portugais de Josué de Castro ont été traduites par l'auteur du présent texte.

10 Ibid., p. 15.
}

sur les tabous alimentaires ${ }^{11}$. Ce travail repose sur un recueil de données de terrain d'où il ressort qu'un grand nombre de restrictions d'usage des aliments, fondées sur de pures superstitions, ont cours pendant certaines périodes de $l^{\prime}$ année ${ }^{12}$. Il propose une interprétation de ces tabous en les mettant en rapport avec les différents modes d'appropriation de la terre et des ressources naturelles et, surtout, avec les inégalités sociales dont ils sont la source. Certains sont, selon lui, diffusés au sein de la population par les propriétaires des usines à sucre pour protéger leurs intérêts. C'est le cas, par exemple, des interdictions portant sur les fruits, qui ont comme objectif de leur permettre de s'en réserver l'exclusivité. Il en va de même, beaucoup plus largement, de toutes celles qui ont trait aux cultures vivrières : il faut maintenir sous dépendance alimentaire les populations qui fournissent une main-d'œuvre bon marché, en faisant en sorte qu'elles n'aient pas recours aux produits de cueillette ni aux jardins potagers en forêt - sans compter, à propos de ces derniers, l'objectif de réserver à la canne à sucre la surface forestière disponible.

Ces interdits sont directement des facteurs de sousalimentation. Ils le sont en outre indirectement en perturbant les modes traditionnels d'alimentation des populations. Utilisant ses connaissances agronomiques, Josué de Castro se livre à une critique approfondie de la substitution de la monoculture implantée par les colonisateurs aux pratiques agricoles de polyculture et de culture sur brûlis héritées des Indiens et des Africains. «Le plus grave [...], ce ne fut pas la destruction des aliments utiles à l'alimentation régionale - richesse de la flore, de la faune, du sol-que les Indiens et les esclaves africains connaissaient et dont ils savaient utiliser les propriétés, mais essentiellement la destruction de la polyculture, de l'agriculture de subsistance qu'ils pratiquaient ${ }^{13}$.» La monoculture de la canne remettait aussi en cause les savoirs populaires portant sur les effets bénéfiques des « aliments-médicaments » utilisés par les populations locales et ignorés par la science, dont Josué de Castro souligne l'importance pour l'équilibre alimentaire.

Ce mixage d'anthropologie, d'écologie, de sociologie, de géographie et de sciences de la nutrition fait de lui un des maîtres penseurs d'une analyse interdisciplinaire des relations sociétés-natures, dont la leçon est plus que jamais actuelle.

\footnotetext{
11 Cf. Castro, J. de, 1939. Fisiologia dos tabus, Rio de Janeiro, Editôra Nestlé.

12 Exemples : manger du sucre le matin provoque l'apparition de lombrics dans l'intestin, sucer des bonbons entraîne l'apparition de vers parasites dans le ventre, la mangue avalée avec du lait devient un poison, manger de la pastèque qui vient d'être cueillie dans les broussailles provoque de la fièvre, le fruit peu mûr provoque des coliques, manger la canne verte donne des inflammations aux femmes, les feuilles et les légumes sont des aliments pour les chenilles.

13 Castro, J. de, Geografia da fome, op. cit., p. 107.
} 


\section{Un chercheur citoyen}

Josué de Castro est le contre-modèle du chercheur solitaire. Homme de grande érudition, il a toujours tenu le plus grand compte, dans ses études, des apports des savants de son époque. Il aimait dire que son œuvre s'enrichissait des contributions de divers spécialistes et qu'elle était finalement un produit collectif.

Par ailleurs, il choisissait ses approches et ses méthodes et construisait ses problématiques en tenant compte de la réalité des besoins des populations les plus démunies, sujet de ses recherches. Pour lui, les résultats scientifiques étaient une "arme contre la faim». Quelle que soit la question traitée, il faisait toujours référence à la fonction critique de l'intellectuel. «La critique honnête apporte plus de satisfaction que de contrariétés ${ }^{14}{ }$, écrivit-il.

Cette attitude le conduisit à saisir avec une acuité particulière le phénomène de la sous-alimentation. Il refusait de limiter les études sur ce sujet aux régions d'extrême misère et montrait l'intérêt de prendre en considération les cas où il s'agit d'un phénomène touchant de grandes masses de population, que ce soit de façon endémique (un manque permanent de certains aliments étant responsable de la mort lente des populations) ou épidémique (par exemple, lors d'une sécheresse). Aussi fut-il à l'origine de la reconnaissance et de l'adoption par la FAO des nouveaux concepts de « faim partielle » et de "faim occulte», alors que cette institution n'employait que le terme général de sous-nutrition. Pour faire admettre ses idées, il acceptait les responsabilités du plus haut niveau. Il fut délégué du Brésil à la FAO. Signe de sa réputation internationale acquise auprès des nombreux États qui avaient sollicité son expertise sur les problèmes nutritionnels, il devint le président de cette organisation (de 1952 à 1956).

En mettant en évidence le fait que la faim est moins conditionnée par les facteurs naturels et géographiques que par ceux de nature économique et sociale, il fut un penseur politique du développement. Cette interprétation de la sous-nutrition semble aller de soi. Et pourtant, elle allait à l'encontre des idées courantes et des politiques suivies, en particulier s'agissant du Nordeste brésilien. Il s'accorde avec Aluízio Campos, qu'il cite : « La lutte contre la faim dans le Nordeste ne doit pas être envisagée en termes simplistes de lutte contre la sécheresse, et moins encore contre ses effets, mais en termes de lutte contre le sous-développement régional, expression de la concentration de la terre, de la monoculture, de la structure agraire moyenâgeuse et de la sous-capitalisation de l'exploitation des ressources naturelles ${ }^{15}$. » Mais il est politiquement plus facile et moins risqué pour les

\footnotetext{
14 Ibid., p. 303.

15 Campos, A., 1956. Realidade Econômica e Planejamento do Nordeste, Fortaleza, A.B. Fontenele. Cité dans Castro, J. de, Geografia da fome, op. cit., p. 243.
}

gouvernements de «lutter contre la sécheresse », ce qui fut l'objectif constant des politiques suivies pour le Nordeste (notamment en créant des retenues d'eau, les açudes), que de se lancer dans une réforme agraire, opération politiquement radicale et pourtant seule façon de remédier au sous-développement régional. Le paradoxe, d'ailleurs mais c'était aussi le résultat inévitable de cette politique -, est que lesdits açudes ont davantage servi les intérêts de la classe dominante que ceux des populations pauvres auxquelles l'eau qu'ils retenaient était destinée.

À l'occasion de la première Conférence des Nations unies sur l'environnement et le développement (Stockholm, 1972), Josué de Castro s'est opposé vigoureusement aux thèses néomalthusiennes qui rendent la surpopulation, c'est-à-dire les affamés eux-mêmes, responsable de la faim, alors que, pour lui, c'est l'existence même de la faim qui est la cause de la surpopulation. Et, à ce propos, il reprend le dicton populaire : « La table du pauvre est maigre, mais le lit de la misère est fécond ${ }^{16}$. »

En 1965, date de la publication de la neuvième édition de Géographie de la faim, une nouvelle politique de développement au Brésil voyait le jour. Jusque-là fondée sur l'exportation de matières premières (coton, sucre, café, bois, minerais), elle était graduellement orientée vers une industrialisation à marche forcée, concentrée dans la région sud du Brésil. Les flux migratoires du Nordeste vers le Sud se sont alors renforcés, la main-d'œuvre peu chère étant absorbée par le secteur industriel. Pour Josué de Castro, il s'agissait là d'une forme de développement régional inégal, car, dans le Nordeste, l'économie fondée sur la canne à sucre ne permettait pas un tel essor industriel. Il affirmait qu'il était tout à fait possible de faire prévaloir d'autres évolutions en combinant développement agricole et industriel dans le Nordeste même. Cela, bien sûr, supposait d'autres choix dans la répartition des investissements entre les régions. Et ces choix avaient un sérieux coût politique, car ils supposaient d'entrer en conflit avec la classe des grands propriétaires fonciers, maîtres de la canne à sucre.

Il considérait de son devoir d'assumer politiquement les idées qu'il défendait sur les bases de ses analyses. C'est pourquoi il brigua des mandats électoraux devant lui permettre de lutter politiquement pour cette alternative en faveur du Nordeste au niveau fédéral. Il fut élu une première fois, en 1954, en tête de liste avec Francisco Julião, leader des Ligues paysannes, et réélu en 1957 avec une majorité écrasante de voix dans toute la région du Nordeste. Dans un contexte politique de pressions populaires, il s'est identifié aux revendications radicales des paysans et des travailleurs. Il fut un inspirateur précoce du mouvement actuel des paysans sans terre. Il concevait comme devant aller de pair le développement

\footnotetext{
16 Cité dans Castro, J. de, 1951. Geopolítica da fome, Rio de
} Janeiro, Livraria-Editôra da Casa do Estudante do Brasil. 
agricole, celui du monde rural et celui du monde industriel. Pour lui, la voie à suivre était celle de la réforme agraire : «Toutes les mesures et toutes les initiatives ne seront que des palliatifs pour lutter contre la faim, si on ne procède pas à une réforme agraire nationale qui libère les populations de la servitude de la terre et dont le résultat réponde aux besoins de la grande majorité des familles paysannes. Il en découlerait une création d'emplois qui contribuerait à résorber fortement la famine récurrente. Une telle réforme aurait notamment permis d'éviter les grandes migrations du monde rural du Nordeste vers les grands centres urbains du Sud, provoquées par la faim et le manque de travail ${ }^{17}$.»

Josué de Castro était un scientifique engagé. Dans ses enseignements donnés à Vincennes, il abordait les questions d'actualité les plus vives. Il a aidé un groupe d'enseignants à créer un cursus d'écologie humaine conçu dans une dimension à la fois scientifique et politique. Après une introduction des notions et des concepts de la science écologique, l'enseignement consistait en une étude de cas centrée sur les relations entre les milieux et les sociétés. C'est ainsi que cette équipe a mis en évidence les conséquences désastreuses des pratiques utilisées pendant la guerre du Vietnam, à savoir la diffusion massive de la dioxine (aussi connue sous le nom $\mathrm{d}^{\prime}$ « agent orange »), que ce soit sur l'écosystème forestier tropical, quand elle était utilisée pour défolier entièrement les arbres afin de rendre visibles les cibles humaines des bombardements, ou sur la santé des populations (y compris à long terme à travers ses effets sur les fœtus), quand elle polluait les rizières, source quasiment exclusive de leur alimentation.
Sa réputation internationale, ses idées et sa pratique citoyenne ne pouvaient manquer d'être insupportables pour la dictature militaire au Brésil. C'est pourquoi il fut déchu de ses droits civiques lors du coup d'État de 1964. Cette sanction et l'exil en France qui s'ensuivit expriment plus que tout la force de cette personnalité pour qui la connaissance était une arme de combat et l'académisme, une science morte.

\section{Remerciements}

L'auteur remercie Marcel Jollivet pour l'aide qu'il lui a apportée dans la rédaction de ce texte.

Brésilienne, professeur de biologie, Magda Zanoni est arrivée en France en 1968 avec une bourse du gouvernement français. Contrainte de s'exiler pour des raisons politiques, elle est alors invitée par Josué de Castro à travailler avec lui au Centre universitaire expérimental de Vincennes (Paris 8). Devenue maître de conférences à l'Université Paris Diderot, elle s'est donné comme thème de travail l'approche interdisciplinaire des rapports entre environnement et développement dans les milieux de forêt tropicale humide, de mangrove et de plaines agricoles. Elle a animé ce thème de recherche dans le cadre de la coopération universitaire France-Brésil et participé à la création d'un doctorat interdisciplinaire en environnement et développement et à la mise en place d'une chaire Unesco du développement durable à l'Université fédérale du Parana. Elle est actuellement en délégation au Centre d'études sur le développement agraire du ministère du Développement agraire à Brasilia.

17 Castro, J. de, Geografia da fome, op. cit., pp. 243-244. 